\title{
Composición de Ñames Frescos Cultivados en Colombia y Sometidos a Freído por Inmersión
}

\author{
Armando Alvis ${ }^{1}$, Carlos A. Vélez ${ }^{2}$ Maite Rada-Mendoza ${ }^{3^{*}}$ \\ (1) Universidad de Córdoba, Facultad de Ciencias Agrícolas, Departamento de Ingeniería de \\ Alimentos, Carrera 6 No. 76-103, Km 3, vía Cereté, Córdoba-Colombia \\ (2) Universidad del Valle, Facultad de Ingeniería, Departamento de Ingeniería de Alimentos, \\ Apartado Aéreo 25360, Cali-Colombia \\ (3) Universidad del Cauca, Facultad de Ciencias Naturales, Exactas y de la Educación, \\ Departamento de Química, Calle 5 No. 4-70, Popayán, Cauca-Colombia \\ (e-mail: mrada@unicauca.edu.co)
}

* autor a quien debe ser dirigida la correspondencia

\section{Resumen}

Se determinó la composición proximal de cuatro variedades de ñame en fresco, de harina y de almidón de ñame, y se aportó información sobre la composición en agua y grasa de dos variedades de ñame cocidos por proceso de freído por inmersión. Previamente a las determinaciones, las muestras fueron secadas hasta peso constante. El ñame fresco estuvo compuesto fundamentalmente por agua y carbohidratos, pero fue bajo en proteína, fibra y grasa. En los tres productos estudiados existió una gran diferencia en los contenidos de humedad y proteína entre las variedades estudiadas. La composición de estas variedades producidas en Colombia es similar a las producidas en otros países de Latinoamérica. El ñame es una fuente moderada de energía y por lo tanto, es considerado un alimento valioso en países con problemática de salud nutricional, y se debería promover su consumo.

\section{Composition of Fresh Colombian Yam and Cooked by Deep-Fat Frying}

\begin{abstract}
The proximal composition of four varieties of fresh, flour and starch of the yam was evaluated and water and fat content information content in two yam varieties put under deep fat frying were determined. Before the experiments, the samples were dried until they reached constant weight. The fresh yam consists mainly of water and carbohydrates, with low levels of protein, fiber and fat. High differences were found among varieties for the moisture and protein contents in the products studied. The composition of these yam varieties produced in Colombia are similar to those from other Latin American countries. Yam is a source of moderate energy and therefore is a valuable food in countries with critical nutritional health, and its consumption should be promoted.
\end{abstract}

Keywords: yam, proximal composition, starch, deep-fat frying 


\section{INTRODUCCIÓN}

El ñame (Dioscorea alata), debido a la cantidad de carbohidratos que proporciona, es un alimento básico para muchos de los pobladores de las regiones tropicales y subtropicales húmedas del occidente de África, Asia y el Caribe (Cooursey y Haynes, 1970; Bradbury y Holloway, 1987; ONU, 1990; FAO/FAOSTAT, 2002); en esta última región, en la Costa Atlántica Colombiana (zona de mayor producción de ñame), se cultivan alrededor de 29.757 ha, que involucran a 9.000 familias de pequeños productores, con un área promedio de 1,4 ha por familia, un rendimiento promedio de 11,9 Ton/ha y una producción de 264.500 Ton/año (Minagricultura, 2003).

El sistema de comercialización de los pequeños productores, se caracteriza por bajos volúmenes, escasa infraestructura de acopio, de transporte, de almacenamiento, de transformación y un alto grado de intermediación, que lo hace complejo por la falta de organización de los productores (Bradbury y Holloway, 1987; ONU, 1990); además, la estacionalidad de la producción, hace que exista una sobreoferta en determinada época del año, lo que se refleja en la reducción de precios, caída de la demanda, pérdidas poscosecha (30 a $40 \%$ ) e incapacidad para transformar el producto. En Colombia, donde el $78 \%$ de la producción se dirige al mercado en fresco, no se conocen transformaciones tecnológicas (Rodríguez, 1996), mientras que en África sólo es tradicional la preparación de harina y en los últimos años se comenzó a transformar en chips. Por otra parte, no ha existido interés por parte de los investigadores en conocer las propiedades nutritivas del ñame; no fue si no hasta hace unos pocos años, que en Latinoamérica se comenzó a disponer de datos de valor nutritivo de raíces y tubérculos tropicales crudos y cocidos y por tanto, existe muy poca documentación en tablas o en documentos específicos (ONU, 1990; Wanasundera y Ravindran, 1994; FAO/LATINFOODS, 2002; USP, 2005).

En Colombia, el ñame se consume tradicionalmente en forma de sopa ó sancocho, en el cual se mezcla con yuca, plátano y carne de res o de gallina; combinada con queso, cuajada o suero de leche para preparar el "mote de queso"; cocido, como acompañante de carnes y pescado; en Semana Santa, en la preparación doméstica de tortas y dulces. Como utilización industrial, se reporta la extracción de la Diosgenina, una sapogenina usada como precursor en la síntesis de esteroides (Fang y Kong, 2002); sin embargo, estas utilizaciones no se han realizado comercialmente, ya que países como Nigeria y otros del continente africano, están en capacidad de ofrecer mayores volúmenes y con menores costos (CORPOICA, 1997). Su posible utilización en la fabricación de biocombustibles, ha comenzado a discutirse recientemente; aparte del potencial de la harina y de los productos derivados del ñame, éste se puede convertir en almidón (Ezeh, 1992), principal componente de los rizomas, con un $75 \%$ de materia seca (Moorthy y Nair, 1989).

El componente principal que caracteriza a este tubérculo, es el contenido de carbohidratos complejos en forma de almidón, cuyo consumo se está impulsando en los países más pobres, para mejorar el estado nutricional, para el manejo de la diabetes mellitus y de la obesidad (Jenkins et al., 1981; Hill, 2000); este almidón es estable a alta temperatura y bajo $\mathrm{pH}$, presenta poca cantidad de proteínas y lípidos pero mayor de vitaminas, y es muy rico en minerales (Lasztity et al., 1998).

El presente estudio tuvo como finalidad determinar la composición de cuatro de las variedades de ñames frescos más cultivados en Colombia, utilizando métodos químicos y enzimáticos, además de aportar información sobre la composición de ñames cocidos por proceso de freído por inmersión, con el propósito final de transferir conocimiento nutricional a la población, que permita también incentivar la promoción de su consumo, comercialización, y su incorporación en la tabla de composición de alimentos de América Latina (FAO/LATINFOODS).

\section{MATERIALES Y MÉTODOS}

\section{Muestreo}

Se seleccionaron rizomas de ñame criollo (Dioscorea alata), los cuales fueron adquiridos a través del Banco de Germoplasma de la Universidad de Córdoba (Córdoba-Colombia) y pertenecientes a la región de la Costa Atlántica. De cada variedad se recolectaron $10 \mathrm{Kg}$ de muestra de primera calidad y de 100 a $120 \mathrm{~g}$ de peso por unidad. El ñame recolectado en forma manual y de acuerdo a su número 
de parcela, fue de las variedades Bolañero, Ecuatoriano, Pico de Botella y Diamante 22, que son los producidos en mayor cantidad en el país.

A las muestras recién recolectadas y trasladadas al laboratorio, se les determinó el peso unitario con una balanza granataria, pesando de cada variedad, ocho unidades de muestra fresca y cruda.

\section{Preparación de la muestra}

Todas las unidades de cada muestra fueron lavadas, peladas y cortadas con un "potato-cutter" en forma de paralelepípedo de aproximadamente $0,8 \times 0,8 \times 4 \mathrm{~cm}$ de longitud y se conformó una muestra compuesta que se almacenó en refrigeración durante un tiempo máximo de tres días. Previamente a los análisis, la muestra fue secada en un horno de vacío de 95 a $97^{\circ} \mathrm{C}$ siguiendo el método número 925.09 de la AOAC (AOAC, 2003).

\section{Caracterización de las muestras}

En la muestra seca, se determinó por triplicado el contenido de grasa, proteína, ceniza y fibra, siguiendo los métodos de la AOAC, números 920.39, 920.87, 923.03 y 962.09, respectivamente (AOAC, 2003). Las muestras restantes se almacenaron durante 90 días a $28^{\circ} \mathrm{C}$.

Los carbohidratos totales se estimaron por diferencia, como se muestra en la ecuación 1.

Carbohidratos totales $=100-(\%$ humedad $+\%$ proteína $+\%$ grasa $+\%$ ceniza $)$

Adicionalmente, se realizaron los análisis por triplicado de carbohidratos complejos tales como: Almidón disponible, según el método de Mestres et al. (1993), y azúcares reductores según el método de Cronin y Smith (1979), en muestras molidas secas y tamizadas a un tamaño de partícula inferior a $300 \mu \mathrm{m}$.

Las calorías fueron calculadas utilizando los factores de conversión de Atwater para los datos derivados del peso fresco como se muestra en la ecuación 2.

Calorías $(\mathrm{kcal} / 100 \mathrm{~g})=(4 \times \%$ proteína $)+(9 \times \%$ grasa $)+(4 \times \%$ carbohidrato $)$

\section{Obtención de almidón nativo y harina}

La extracción del almidón consistió de: lavado, pelado y rallado de los rizomas, extracción por tamizado con agua, sedimentación por centrifugación y secado artificial en horno (Despatch Oven Company) durante 24 horas a $40^{\circ} \mathrm{C}$ hasta obtener una humedad final de 10 a $12 \%$. El almidón obtenido fue empacado en bolsas de polietileno y almacenado a $4^{\circ} \mathrm{C}$.

Para extraer la harina, el procesamiento consistió de: lavado, trozado de los ñames y colocación posterior en una bandeja que se introdujo en un horno de secado durante 24 horas a $40{ }^{\circ} \mathrm{C}$ hasta obtener una humedad de 10 a $12 \%$. Ya seca, se molió y se tamizó con un tamiz a $250 \mu \mathrm{m}$.

La caracterización de las muestras de rizomas, de harina y de almidón de ñame, fue realizada en el laboratorio de Química de la Universidad del Cauca (Popayán-Colombia).

\section{Freído}

El método de cocción elegido fue la fritura por inmersión; para ello, se utilizó aceite de soya (gravedad específica 0,92) en una freidora de acero inoxidable (mkE, USA) de 5 L de capacidad, con sistema de control de temperatura. Las temperaturas $\left(140,160\right.$ y $\left.180{ }^{\circ} \mathrm{C}\right)$ y los tiempos netos de fritura $(120,150$ y $180 \mathrm{~s})$, fueron establecidos mediante pruebas preliminares, ya que bajo estas condiciones, las muestras presentaron una textura apetecible para el gusto local. El producto frito se escurrió, se enfrió y se homogenizó manualmente en un molino de maíz para su análisis inmediato. Se determinó por triplicado la humedad y el contenido de grasa según los métodos 925.09 y 920.39 de la AOAC (2003). 


\section{Estadística}

Los datos se analizaron con el programa estadístico SPSS (versión 10,0) para Windows. La comparación de medias se realizó usando análisis de varianza (ANOVA) y test de comparación múltiple LSD.

\section{RESULTADOS Y DISCUSIÓN}

En la tabla 1 se indica el peso unitario promedio de cada una de las variedades de ñame colombianas en estudio; como se observa, no existen diferencias significativas ( $p \geq 0,01)$ en peso, entre las variedades analizadas. La variabilidad en el peso de una misma variedad resultó baja, hasta en un 5,4 $\%$. Los pesos unitarios hallados, se encuentran muy por debajo de los mínimos reportados por Blanco-Metzler et al. (2004).

La tabla 2 muestra la composición proximal de las cuatro variedades de ñame en fresco. Como se observa, están compuestos en mayor proporción por agua y carbohidratos y por lo tanto, son fuentes moderadas de energía; solo se detectaron niveles traza de grasa, muy inferiores a los reportados por Wanasundera y Ravindran (1992); el contenido en proteína y fibra fue muy inferior a lo reportado por Wanasundera y Ravindran (1992) y Chou et al. (2006), aunque más elevado, comparado con los reportados en la literatura (USP, 2005). Las variedades analizadas, muestran mayor riqueza en minerales que las reportadas por Belitz y Grosch (1997), FAO/LATINFOODS (2002) y USP (2005), aunque sus valores son similares a los reportados por Chou et al. (2006). El contenido de almidón para las cuatro variedades analizadas fue superior a $78,7 \%$, valor reportado por Wanasundera y Ravindran (1992) para tubérculos de Dioscorea alata. Los contenidos de humedad y proteína, son los caracteres para los que existen diferencias significati-vas $(p \leq 0,01)$ entre todas las variedades.

El ñame Pico de Botella fue la variedad con mayor contenido en agua, grasa y fibra pero menor en proteína y cenizas, aunque en composición fue similar a la reportada en la literatura (USP, 2005); desde el punto de vista de manejo dietético, esta variedad es muy interesante, ya que mostró menor contenido energético, al contener más agua.

La tabla de composición de alimentos de América latina (FAO/LATINFOODS, 2002), muestra valores semejantes a los obtenidos aquí, a excepción de los valores de proteína y grasa, que para estas variedades fue mucho menor a las reportadas.

Tabla 1: Peso unitario medio, desviación estándar y coeficiente de variación de cuatro variedades de ñame cultivadas en Colombia. Letras diferentes en una misma línea indican diferencia significativa $(p \leq 0,01)$

\begin{tabular}{|l|c|c|c|c|}
\hline \multicolumn{1}{|c|}{ Estadísticos } & Ecuatoriano & Bolañero & Pico de Botella & Diamante 22 \\
\hline Promedio (g) & $113,5^{\mathrm{a}}$ & $121,9^{\mathrm{a}}$ & $118,8^{\mathrm{a}}$ & $116,6^{\mathrm{a}}$ \\
\hline Desviación estándar & 6,1 & 3,6 & 2,1 & 3,0 \\
\hline Coeficiente de variación (\%) & 5,4 & 3,0 & 1,8 & 2,6 \\
\hline Número de muestras (n) & 8 & 8 & 8 & 8 \\
\hline
\end{tabular}

Tabla 2: Composición promedio ( \pm desviación estándar), en base húmeda $(\mathrm{N}=3)$ de agua, proteína, grasa, cenizas, fibra y carbohidratos de cuatro variedades de ñames cultivadas en Colombia. Letras diferentes en una misma línea indican diferencias significativas $(p \leq 0,01)$.

\begin{tabular}{|l|c|c|c|c|}
\hline \multicolumn{1}{|c|}{ Estadísticos (BH) } & Ecuatoriano & Bolañero & Pico de Botella & Diamante 22 \\
\hline Humedad (\%) & $70,53 \pm 0,11^{\mathrm{c}}$ & $66,22 \pm 0,25^{\mathrm{a}}$ & $74,99 \pm 0,91^{\mathrm{d}}$ & $69,18 \pm 0,59^{\mathrm{b}}$ \\
\hline Proteína (\%) & $1,95 \pm 0,03^{\mathrm{d}}$ & $1,68 \pm 0,01^{\mathrm{b}}$ & $1,48 \pm 0,06^{\mathrm{a}}$ & $1,84 \pm 0,01^{\mathrm{c}}$ \\
\hline Grasa (\%) & $0,09 \pm 0,00^{\mathrm{c}}$ & $0,03 \pm 0,00^{\mathrm{a}}$ & $0,09 \pm 0,00^{\mathrm{c}}$ & $0,04 \pm 0,00^{\mathrm{b}}$ \\
\hline Cenizas (\%) & $1,19 \pm 0,01^{\mathrm{c}}$ & $1,12 \pm 0,01^{\mathrm{b}}$ & $0,99 \pm 0,04^{\mathrm{a}}$ & $1,01 \pm 0,08^{\mathrm{a}}$ \\
\hline Fibra (\%) & $0,06 \pm 0,00^{\mathrm{a}}$ & $0,06 \pm 0,00^{\mathrm{a}}$ & $0,21 \pm 0,00^{\mathrm{c}}$ & $0,10 \pm 0,00^{\mathrm{b}}$ \\
\hline Carbohidratos totales (\%) & 26,24 & 30,95 & 22,45 & 27,93 \\
\hline Energía (kcal) & 113,57 & 130,79 & 96,53 & 112,08 \\
\hline Almidón (\%) & 83,3 & 89,0 & 83,3 & 90,0 \\
\hline
\end{tabular}


El contenido en macronutrientes para las harinas se resume en la tabla 3. La composición mayor es en carbohidratos y agua y con base en esa proporción, se consideran buenas fuentes de energía. El contenido de proteína y cenizas es alto. Pico de Botella presentó un contenido significativamente mayor de cenizas, que el resto de las variedades. Los contenidos de humedad, proteína y grasa, son los caracteres para los que existen diferencias significativas $(p \leq 0,01)$ entre todas las variedades.

Para la obtención de harina, la variedad Pico de Botella es mejor por color, ya que no se pardea, por lo que permite la obtención de una harina blanca de buena calidad; sin embargo, en cuanto a rendimiento la variedad que da mejor resultado es Bolañero, pero tiene la desventaja de que se pardea más rápidamente.

En la tabla 4 se indica el contenido en macronutrientes de las cuatro variedades de ñame en forma de almidones, los cuales están compuestos en mayor cantidad por agua y carbohidratos; proporcionan más energía que los productos frescos y en forma de harinas ya que al contener menos humedad, concentran los sólidos incrementando así su valor energético. La humedad encontrada en los cuatro almidones analizados, concuerda con la información reportada en la literatura para almidón nativo de ñame, el cual varió de 10,00 a 10,40 \% (Jinsong et al., 2006). El contenido en proteína fue similar al hallado por Alves et al. (1999), quienes reportaron valores de 0,20\% y por Rodríguez (1996), quien reportó valores de 0,5 \% para almidones de ñame criollo y espino y aunque todas las variedades difieren significativamente entre sí, el contenido proteico es bajo en todas ellas, al igual que las cenizas; sin embargo, la cantidad de cenizas se relaciona posiblemente con un aumento en el contenido de minerales en los almidones, resultado similar al encontrado por Hoover (2002), quien indicó que el fósforo y otros minerales, influyen en el contenido final de las cenizas. Los valores de cenizas hallados para las cuatro variedades de almidón de ñame, fueron superiores a los reportados por Alves et al. (1999); la variedad Pico de Botella presentó un contenido significativamente superior, comparado con el resto de las variedades. Los valores de fibra fueron iguales a los reportados en almidones de ñame criollo y espino por Rodríguez (1996). Los contenidos en grasa concuerdan con lo reportado por Hoover (2002) en almidones de ñame $(0,00$ a 0,05\%), pero son inferiores a los reportados por Alves et al. (1999) (0,15\%).

Tabla 3: Composición promedio ( \pm desviación estándar), en base húmeda $(\mathrm{N}=3)$ de agua, proteína, grasa, cenizas, fibra y carbohidratos de harinas de cuatro variedades de ñames cultivadas en Colombia. Letras diferentes en una misma línea indican diferencias significativas $(p \leq 0,01)$.

\begin{tabular}{|l|c|c|c|c|}
\hline \multicolumn{1}{|c|}{ Estadísticos (BH) } & Ecuatoriano & Bolañero & Pico de Botella & Diamante 22 \\
\hline Humedad (\%) & $12,19 \pm 0,03^{\mathrm{a}}$ & $18,26 \pm 0,04^{\mathrm{d}}$ & $16,68 \pm 0,15^{\mathrm{b}}$ & $17,99 \pm 0,00^{\mathrm{c}}$ \\
\hline Proteína (\%) & $6,15 \pm 0,00^{\mathrm{d}}$ & $5,13 \pm 0,33^{\mathrm{b}}$ & $6,07 \pm 0,04^{\mathrm{c}}$ & $4,67 \pm 0,00^{\mathrm{a}}$ \\
\hline Grasa (\%) & $0,07 \pm 0,00^{\mathrm{c}}$ & $0,05 \pm 0,00^{\mathrm{a}}$ & $0,06 \pm 0,00^{\mathrm{b}}$ & $0,15 \pm 0,02^{\mathrm{d}}$ \\
\hline Cenizas (\%) & $3,17 \pm 0,25^{\mathrm{a}}$ & $3,05 \pm 0,01^{\mathrm{a}}$ & $3,64 \pm 0,12^{\mathrm{b}}$ & $3,05 \pm 0,00^{\mathrm{a}}$ \\
\hline Fibra (\%) & $0,11 \pm 0,01^{\mathrm{a}}$ & $0,10 \pm 0,00^{\mathrm{a}}$ & $0,11 \pm 0,00^{\mathrm{a}}$ & $0,16 \pm 0,01^{\mathrm{b}}$ \\
\hline Carbohidratos totales (\%) & 78,42 & 73,51 & 73,55 & 74,14 \\
\hline Energía (kcal) & 338,91 & 315,01 & 319,02 & 316,59 \\
\hline
\end{tabular}

Las variedades Pico de Botella y Diamante 22, fueron las muestras seleccionadas para ser sometidas al proceso de fritura, ya que fueron las que presentaron las mejores características en cuanto a contenido en azúcares reductores $(0,20 \pm 0,02$ y 0,25 $\pm 0,04$, respectivamente) y al dejarlas al medio ambiente, eran las que tardaban más en sufrir pardeamiento enzimático (Alvis y Vélez, 2006). El método de fritura por inmersión fue seleccionado, debido a que es un método rápido de cocción y el alimento (en este caso el ñame), se cuece uniformemente por todos los lados (Rahman, 2003).

En la tabla 5 se muestra el contenido en agua y grasa para los ñames fritos a 140,160 y $180{ }^{\circ} \mathrm{C}$, durante 120, 150 y $180 \mathrm{~s}$, es decir, con un nivel de cocción comestible y apetecible sensorialmente para el consumidor. Las muestras control, son aquellas que no fueron sometidas al proceso de fritura. Como era de esperar en las muestras fritas de ambas variedades, con el incremento de la temperatura y del tiempo de fritura, la humedad disminuyó, mientras que la grasa aumentó. Comparando estos valores con los reportados en la literatura por Blanco-Metzler et al. (2004), en muestras de ñame cocidas, los contenidos de humedad fueron más bajos y los de grasa más elevados para estas variedades. 
Tabla 4: Composición promedio ( \pm desviación estándar), en base húmeda $(\mathrm{N}=3)$ de agua, proteína, grasa, cenizas, fibra y carbohidratos de almidones nativos de cuatro variedades de ñames cultivadas en Colombia. Letras diferentes en una misma línea indican diferencias significativas $(p \leq 0,01)$.

\begin{tabular}{|l|c|c|c|c|}
\hline \multicolumn{1}{|c|}{ Estadísticos (BH) } & Ecuatoriano & Bolañero & Pico de Botella & Diamante 22 \\
\hline Humedad (\%) & $8,66 \pm 0,05^{\mathrm{a}}$ & $9,75 \pm 0,07^{\mathrm{b}}$ & $10,22 \pm 0,04^{\mathrm{c}}$ & $10,09 \pm 0,11^{\mathrm{c}}$ \\
\hline Proteína (\%) & $0,13 \pm 0,01^{\mathrm{b}}$ & $0,10 \pm 0,00^{\mathrm{a}}$ & $0,49 \pm 0,01^{\mathrm{d}}$ & $0,28 \pm 0,01^{\mathrm{c}}$ \\
\hline Grasa (\%) & $0,06 \pm 0,01^{\mathrm{b}}$ & $0,00 \pm 0,00^{\mathrm{a}}$ & $0,06 \pm 0,00^{\mathrm{b}}$ & $0,00 \pm 0,00^{\mathrm{a}}$ \\
\hline Cenizas (\%) & $0,45 \pm 0,03^{\mathrm{a}}$ & $0,39 \pm 0,01^{\mathrm{a}}$ & $0,69 \pm 0,04^{\mathrm{b}}$ & $0,36 \pm 0,06^{\mathrm{a}}$ \\
\hline Fibra (\%) & $0,00 \pm 0,00^{\mathrm{a}}$ & $0,00 \pm 0,00^{\mathrm{a}}$ & $0,00 \pm 0,00^{\mathrm{a}}$ & $0,00 \pm 0,00^{\mathrm{a}}$ \\
\hline Carbohidratos totales (\%) & 90,7 & 89,76 & 88,54 & 89,27 \\
\hline Energía (kcal) & 363,86 & 359,44 & 356,66 & 358,2 \\
\hline
\end{tabular}

En la tabla 6 se muestran los valores correspondientes a la pérdida de agua y ganancia de grasa de las variedades fritas. Como se observa, a medida que se aumentó la temperatura y el tiempo de fritura, estas variedades contienen menos agua, pero más grasa que las muestras crudas; con base en estas pérdidas, se deduce que la variedad Diamante 22, es la que pierde más agua y por ende gana más grasa en las condiciones más extremas de tiempo y temperatura de fritura. Comparando la ganancia en grasa para las variedades Pico de Botella y Diamante 22 a $140{ }^{\circ} \mathrm{C}$, se observó que a tiempos de fritura de 120 y $150 \mathrm{~s}$, no existen diferencias significativas $(p \leq 0,01)$ entre ambas variedades.

En general, se observa que el contenido de agua disminuye debido a su evaporación durante el freído. Esta humedad es gradualmente reemplazada con aceite y por lo tanto, el contenido en este incrementa con el tiempo de fritura. La disminución en humedad y el incremento en aceite fue más rápido al comienzo de la fritura, pero luego disminuyó lentamente hasta que la fritura terminó, debido a la disminución en la fuerza impulsora de secado (Sahin et al., 1999).

Tabla 5: Composición promedio, en base húmeda $(\mathrm{N}=3)$ de agua y grasa de las variedades Pico de Botella y Diamante 22, sometidas a fritura.

\begin{tabular}{|c|c|c|c|c|c|c|c|c|c|}
\hline Variedad & $\begin{array}{c}\text { Temperatura } \\
\left({ }^{\circ} \mathrm{C}\right)\end{array}$ & $\begin{array}{c}\text { Control } \\
\text { Humedad (\%) }\end{array}$ & \multicolumn{3}{|c|}{ Humedad (\%) } & \multicolumn{3}{c|}{$\begin{array}{c}\text { Control } \\
\text { Grasa (\%) }\end{array}$} & \multicolumn{3}{|c|}{ Grasa (\%) } \\
\hline & & & \multicolumn{3}{|c|}{ Tiempo de fritura } & & \multicolumn{3}{c|}{ Tiempo de fritura } \\
\hline & & & $120 \mathrm{~s}$ & $150 \mathrm{~s}$ & $180 \mathrm{~s}$ & & $120 \mathrm{~s}$ & $150 \mathrm{~s}$ & $180 \mathrm{~s}$ \\
\hline \multirow{3}{*}{$\begin{array}{c}\text { Pico de } \\
\text { Botella }\end{array}$} & 140 & 74,99 & 62,79 & 61,83 & 59,96 & 0,09 & 3,28 & 3,29 & 4,21 \\
\cline { 2 - 11 } & 160 & 74,99 & 61,28 & 58,31 & 57,74 & 0,09 & 3,63 & 4,15 & 4,26 \\
\cline { 2 - 11 } & 180 & 74,99 & 57,64 & 52,76 & 47,67 & 0,09 & 4,41 & 4,77 & 4,88 \\
\hline \multirow{2}{*}{$\begin{array}{c}\text { Diamante } \\
22\end{array}$} & 140 & 69,18 & 54,30 & 52,63 & 48,37 & 0,04 & 3,29 & 3,26 & 3,32 \\
\cline { 2 - 11 } & 160 & 69,18 & 52,78 & 48,53 & 45,22 & 0,04 & 3,52 & 4,75 & 4,94 \\
\cline { 2 - 11 } & 180 & 69,18 & 46,19 & 38,45 & 32,84 & 0,04 & 3,67 & 5,26 & 7,69 \\
\hline
\end{tabular}

Tabla 6: Composición promedio de pérdida de agua y ganancia de grasa de las variedades Pico de Botella y Diamante 22, sometidas a fritura.

\begin{tabular}{|c|c|c|c|c|c|c|c|}
\hline Variedad & $\begin{array}{c}\text { Temperatura } \\
\left({ }^{\circ} \mathrm{C}\right)\end{array}$ & \multicolumn{3}{|c|}{ Pérdida de agua (\%) } & \multicolumn{3}{c|}{ Ganancia de grasa (\%) } \\
\hline & & \multicolumn{3}{|c|}{ Tiempo de fritura } & \multicolumn{3}{c|}{ Tiempo de fritura } \\
\hline & & $120 \mathrm{~s}$ & $150 \mathrm{~s}$ & $180 \mathrm{~s}$ & $120 \mathrm{~s}$ & $150 \mathrm{~s}$ & $180 \mathrm{~s}$ \\
\hline \multirow{3}{*}{$\begin{array}{c}\text { Pico de } \\
\text { Botella }\end{array}$} & 140 & 12,20 & 13,16 & 15,03 & 3,19 & 3,20 & 4,12 \\
\cline { 2 - 8 } & 160 & 13,71 & 16,68 & 17,25 & 3,54 & 4,06 & 4,17 \\
\cline { 2 - 8 } & 180 & 17,35 & 22,23 & 27,32 & 4,32 & 4,68 & 4,79 \\
\hline \multirow{2}{*}{$\begin{array}{c}\text { Diamante } \\
22\end{array}$} & 140 & 14,88 & 16,55 & 20,81 & 3,25 & 3,22 & 3,28 \\
\cline { 2 - 8 } & 160 & 16,40 & 20,65 & 23,96 & 3,48 & 4,71 & 4,90 \\
\cline { 2 - 7 } & 180 & 22,99 & 30,73 & 36,34 & 3,63 & 5,22 & 7,65 \\
\hline
\end{tabular}

Pico de Botella es la variedad recomendada para ser sometida al proceso de fritura, debido a que es la que tiene la menor cantidad de azúcares reductores y se demora más en pardearse. Estos azúcares son los causantes del pardeamiento durante el freído, debido a su reacción con el grupo 
amino de las proteínas; por tanto, juegan un papel importante al decidir el tiempo de fritura, el contenido de humedad y de aceite, el color y la calidad del producto. La variedad Diamante 22, aunque reúne las condiciones anteriores, tiene la desventaja de que se pardea más rápido.

Los resultados indicaron que la temperatura ideal de fritura para el paralelepípedo de ñame es de $180{ }^{\circ} \mathrm{C}$ durante un tiempo de $180 \mathrm{~s}$ en términos de pérdida de agua y apariencia del ñame frito; esta da una mejor costra, un mejor color, mejor textura y menor absorción de aceite.

\section{CONCLUSIONES}

Las cuatro variedades de ñame analizadas en fresco, en harina y en almidón, están compuestas principalmente por agua y carbohidratos, y por tanto, son nutrientes básicos, de gran importancia en la alimentación humana. En cuanto a rendimiento, la variedad Bolañero es la que permite una mayor obtención de harina, aunque tiene la desventaja de que se pardea más rápidamente. La variedad Diamante 22, es la que permite una mayor obtención de almidón, cuyo consumo se puede impulsar tanto a nivel nacional como internacional. Pico de Botella, es la variedad que por sus propiedades, es recomendada para someterla al proceso de fritura, a una temperatura de $180^{\circ} \mathrm{C}$ durante $180 \mathrm{~s}$.

\section{AGRADECIMIENTOS}

A la Universidad del Cauca, por los análisis proximales; al CIAT de Palmira, por la extracción de almidón y harina y por los análisis de azúcares, almidón y materia seca.

\section{REFERENCIAS}

Alves, R.M.L., M.V.E. Grossmann y R.S.S.F. Silva; Gelling properties of extruded yam (Dioscorea alata) starch, Food Chemistry: 67(2), 123-127 (1999).

Alvis, A. y C. Vélez; Determinación del coeficiente de transferencia de calor del ñame (Dioscorea alata) durante un tratamiento térmico por inmersión, Propuesta Doctoral, Dpto. Ing. Alimentos, Universidad del Valle, Cali, Colombia (2006).

AOAC; Métodos de análisis de la asociación oficial de química analítica para determinar humedad, fibra, cenizas, grasa y proteína, Chapter 32: 1, 2, 5 y 14, Washington, U.S.A. (2003).

Belitz, H.D. y W. Grosch; Química de los alimentos, 2ª edición, 825-832. Acribia, S.A., Zaragoza, España (1997).

Blanco-Metzler, A., J. Tovar y M. Fernández-Piedra; Caracterización nutricional de los carbohidratos y composición centesimal de raíces y tubérculos tropicales cocidos, cultivados en Costa Rica, Archivos latinoamericanos de nutrición: 54(3), 322-327 (2004).

Bradbury, H. y W.D. Holloway; Chemistry of tropical root crops: significance for nutrition and agriculture in the pacific, Monograph $\mathrm{N}^{\circ} 6$, The Australian Centre for International Agricultural Research (ACIAR), 201p, Canberra, Australia (1987).

Chou, Su-Tze y otros cuatro autores; Effects of storage temperatures on the antioxidative activity and composition of yam, Food Chemistry: 98(4), 618-623 (2006).

Cooursey, D.G. y P.H. Haynes; Root crops and their potential as in the tropics, World Crops: 22(4), 261-265 (1970).

CORPOICA; Identificación de posibilidades de acondicionamiento y transformación de raíces y tubérculos autóctonos (1997). http://www.iica.int/prociandino/proyecto frutales exoticos.pdf. Acceso: 4 de septiembre (2007).

Cronin, D.A. y S.A. Smith; Determinación de azúcares reductores y totales: según el método de simple and rapid procedure for the analysis of reducing, total and individual sugars in potatoes, Potato Research: 22(2), 99-105 (1979). 
Ezeh, N.; Economics of yam flour production: implications for research and development, and promotion of yam-based industries in Nigeria, Tropical Agriculture (Trinidad): 69(1), $51-57$ (1992).

Fang, M.C. y R.L. Kong; Immuno-bioactive of yam crude polysaccharide and yam yogurt in cell culture and mice, Annual Meeting of Health Food Society of Taiwan, 66p, Taipei, Taiwan (2002).

FAO/FAOSTAT Agriculture data; Food and agriculture organisation of the United Nations (2002), http://apps.fao.org/collections. Acceso: 17 de junio (2007).

FAO/LATINFOODS; Tabla de composición de alimentos de América Latina (2002), http://www.rlc.fao. org/bases/alimento. Acceso: 29 de agosto (2007).

Hill, J.; Los carbohidratos en el control de peso, Boletín Dieta y Salud, Órgano Informativo de Alimentos Kellogg's sobre la Relación entre la Nutrición y la Salud, N1, 8p (2000).

Hoover, R.; Effect of heat-moisture treatment on the structure and physicochemical properties of tuber and root starches, Carbohidrate Polymer: 49(4), 425-437 (2002).

Jenkins, D.J.A. y otros nueve autores; Glycemic index of foods: a physiological basis for carbohydrate exchange, American Journal of Clinical Nutrition: 34(3), 362-366 (1981).

Jinsong, B., S. Shen., M. Sun y H. Corke; Analysis of genotipic diversity in the starch physicochemical properties of nonwaxy rice: apparent amilose content, pasting viscosity and gel textura, StarchStärke: 58(6), 259-267 (2006).

Lasztity, R., M. Hidvegi y A. Bata; Saponins in food, Food Reviews Internacional: 14(4), 371-390 (1998).

Mestres, C., P. Colonna, M.C. Alexandre y F. Matencio; Determinación de almidón: según el método de comparison of various process for making maize pasta, Journal of Cereal Science: 17(3), 277-290 (1993).

Minagricultura; Ministerio de agricultura y desarrollo rural, Anuario estadístico del sector agropecuario (2003). http://www.agrocadenas.gov.co/lacteos/Documentos/caracterizacion lacteos.pdf. Acceso: 4 de septiembre (2007).

Moorthy, S.N. y S.G. Nair; Studies on Dioscorea rotundata starch properties, Starch-Stärke: 41(3), 81-83 (1989).

ONU; Raíces, tubérculos, plátanos y bananas en la nutrición humana, In Colección FAO: Alimentación y nutrición humana, N 24, 196p, Roma, Italia (1990).

Rahman, M. S.; Manual de Conservación de los alimentos, 173p, Acribia, S.A, España (2003).

Rodríguez, G.; Breve reseña del ñame en Colombia, In Corporación Colombiana de Investigación Agropecuaria (CORPOICA) (1996). http://agronomia.unal.edu.co/documentos/Volumen23-2.pdf. Acceso: 4 de septiembre (2007).

Sahin, S., S.K. Sastry y L. Bayindirli; The determination of convective heat transfer coefficient during frying, Journal of Food Engineering: 39(3), 307-311 (1999).

USP; Universidade de São Paulo, Brasil, Tabela brasileira de composição de alimentos (2005), http://www.fcf.usp.br/tabela/buscar alim.asp. Acceso: 29 de agosto (2007).

Wanasundera, J.P.D. y G. Ravindran; Effects of cooking on the nutrient and antinutrient contents of yam tubers (Dioscorea alata and Dioscorea esculenta), Food Chemistry: 45(4), 247-250 (1992).

Wanasundera, J.P.D. y G. Ravindran; Nutrition assessment of yam (Dioscorea alata) tubers, Plant Foods for Human Nutrition: 46(1), 33-39 (1994). 